CERN-EP/2001-013

1 February 2001

\title{
Search for a fermiophobic Higgs at LEP 2
}

DELPHI Collaboration

\begin{abstract}
Higgs bosons predicted by the fermiophobic scenario within Two Higgs Doublets Models were searched for in the data collected by the DELPHI detector at centre-of-mass energies between $189 \mathrm{GeV}$ and $202 \mathrm{GeV}$, corresponding to a total integrated luminosity of $380 \mathrm{pb}^{-1}$. No signal was found and confidence limits were derived in the framework of possible extensions of the Standard Model Higgs sector.
\end{abstract}


P.Abreu $^{22}$, W.Adam ${ }^{51}$, T.Adye ${ }^{37}$, P.Adzic ${ }^{12}$, I.Ajinenko ${ }^{43}$, Z.Albrecht ${ }^{19}$, T.Alderweireld ${ }^{2}$, G.D.Alekseev ${ }^{18}$, R.Alemany ${ }^{9}$, T.Allmendinger ${ }^{19}$, P.P.Allport ${ }^{23}$, S.Almehed ${ }^{25}$, U.Amaldi ${ }^{29}$, N.Amapane ${ }^{46}$, S.Amato ${ }^{48}$, E.Anashkin ${ }^{36}$, E.G.Anassontzis ${ }^{3}$, P.Andersson $^{45}$, A.Andreazza ${ }^{28}$, S.Andringa ${ }^{22}$, N.Anjos ${ }^{22}$, P.Antilogus ${ }^{26}$, W-D.Apel ${ }^{19}$, Y.Arnoud ${ }^{16}, \quad$ B.Åsman ${ }^{45}$, J-E.Augustin ${ }^{24}$, A.Augustinus ${ }^{9}$, P.Baillon ${ }^{9}$, A.Ballestrero ${ }^{46}$, P.Bambade ${ }^{9,21}$, F.Barao ${ }^{22}$, G.Barbiellini ${ }^{47}$, R.Barbier ${ }^{26}$, D.Y.Bardin ${ }^{18}$, G.Barker ${ }^{19}$, A.Baroncelli ${ }^{39}$, M.Battaglia ${ }^{17}$, M.Baubillier ${ }^{24}$, K-H.Becks ${ }^{53}$, M.Begalli ${ }^{6}$, A.Behrmann ${ }^{53}$, Yu.Belokopytov $^{9}$, N.C.Benekos ${ }^{32}$, A.C.Benvenuti ${ }^{5}$, C.Berat ${ }^{16}$, M.Berggren ${ }^{24}$, L.Berntzon ${ }^{45}$, D.Bertrand ${ }^{2}$, M.Besancon ${ }^{40}$, N.Besson ${ }^{40}$, M.S.Bilenky ${ }^{18}$, D.Bloch ${ }^{10}$, H.M.Blom ${ }^{31}$, L.Bol ${ }^{19}$, M.Bonesini ${ }^{29}$, M.Boonekamp ${ }^{40}$, P.S.L.Booth ${ }^{23}$, G.Borisov $^{21}$, C.Bosio ${ }^{42}$, O.Botner ${ }^{49}$, E.Boudinov ${ }^{31}$, B.Bouquet ${ }^{21}$, T.J.V.Bowcock ${ }^{23}$, I.Boyko ${ }^{18}$, I.Bozovic ${ }^{12}$, M.Bozzo ${ }^{15}$, M.Bracko ${ }^{44}$, P.Branchini ${ }^{39}$, R.A.Brenner ${ }^{49}$, P.Bruckman ${ }^{9}$, J-M.Brunet ${ }^{8}$, L.Bugge ${ }^{33}$, P.Buschmann ${ }^{53}$, M.Caccia ${ }^{28}$, M.Calvi ${ }^{29}$, T.Camporesi ${ }^{9}$, V.Canale ${ }^{38}$, F.Carena ${ }^{9}$, L.Carroll ${ }^{23}$, C.Caso ${ }^{15}$, M.V.Castillo Gimenez ${ }^{50}$, A.Cattai $^{9}$, F.R.Cavallo ${ }^{5}$, M.Chapkin ${ }^{43}$, Ph.Charpentier ${ }^{9}, \quad$ P.Checchia ${ }^{36}$, G.A.Chelkov ${ }^{18}$, R.Chierici ${ }^{46}, \quad$ P.Chliapnikov ${ }^{9,43}$, P.Chochula ${ }^{7}$, V.Chorowicz ${ }^{26}$, J.Chudoba ${ }^{30}$, K.Cieslik ${ }^{20}$, P.Collins ${ }^{9}$, E.Cortina ${ }^{50}$, G.Cosme ${ }^{21}$, F.Cossutti ${ }^{9}$, M.Costa ${ }^{50}$, H.B.Crawley ${ }^{1}$, D.Crennell ${ }^{37}$, J.Croix ${ }^{10}$, G.Crosetti ${ }^{15}$, J.Cuevas Maestro ${ }^{34}$, S.Czellar ${ }^{17}$, J.D'Hondt ${ }^{2}$, J.Dalmau ${ }^{45}$, M.Davenport ${ }^{9}$, W.Da Silva ${ }^{24}$, G.Della Ricca ${ }^{47}$, P.Delpierre ${ }^{27}$, N.Demaria ${ }^{46}$, A.De Angelis ${ }^{47}, \quad$ W.De Boer ${ }^{19}$, C.De Clercq ${ }^{2}$, B.De Lotto ${ }^{4}$, A.De Min ${ }^{9}$, L.De Paula ${ }^{48}$, H.Dijkstra ${ }^{9}$, L.Di Ciaccio ${ }^{38}$, K.Doroba ${ }^{52}$, M.Dracos ${ }^{10}$, J.Drees ${ }^{53}$, M.Dris $^{32}$, G.Eigen ${ }^{4}$, T.Ekelof ${ }^{49}$, M.Ellert ${ }^{49}$, M.Elsing ${ }^{9}$, J-P.Engel ${ }^{10}$, M.Espirito Santo ${ }^{9}$, G.Fanourakis ${ }^{12}$, D.Fassouliotis ${ }^{12}$, M.Feindt ${ }^{19}$, J.Fernandez ${ }^{41}$, A.Ferrer ${ }^{50}$, E.Ferrer-Ribas ${ }^{21}$, F.Ferro ${ }^{15}$, A.Firestone ${ }^{1}$, U.Flagmeyer ${ }^{53}$, H.Foeth ${ }^{9}$, E.Fokitis ${ }^{32}$, F.Fontanelli ${ }^{15}$, B.Franek ${ }^{37}$, A.G.Frodesen ${ }^{4}$, R.Fruhwirth ${ }^{51}$, F.Fulda-Quenzer ${ }^{21}$, J.Fuster ${ }^{50}$, A.Galloni ${ }^{23}$, D.Gamba ${ }^{46}$, S.Gamblin ${ }^{21}$, M.Gandelman ${ }^{48}$, C.Garcia ${ }^{50}$, C.Gaspar ${ }^{9}, \quad$ M.Gaspar ${ }^{48}, \quad$ U.Gasparini $^{36}$, Ph.Gavillet $^{9}$, E.N.Gazis ${ }^{32}$, D.Gele ${ }^{10}$, T.Geralis ${ }^{12}$, N.Ghodbane ${ }^{26}$, I.Gil ${ }^{50}$, F.Glege ${ }^{53}$, R.Gokieli ${ }^{9,52}$, B.Golob ${ }^{9,44}$, G.Gomez-Ceballos ${ }^{41}$, P.Goncalves ${ }^{22}$, I.Gonzalez Caballero ${ }^{41}$, G.Gopal ${ }^{37}$, L.Gorn ${ }^{1}$, Yu.Gouz ${ }^{43}$, V.Gracco ${ }^{15}$, J.Grahl ${ }^{1}$, E.Graziani ${ }^{39}$, G.Grosdidier ${ }^{21}$, K.Grzelak ${ }^{52}$, J.Guy ${ }^{37}$, C.Haag ${ }^{19}$, F.Hahn ${ }^{9}$, S.Hahn ${ }^{53}$, S.Haider ${ }^{9}$, A.Hallgren ${ }^{49}$, K.Hamacher ${ }^{53}$, J.Hansen ${ }^{33}$, F.J.Harris ${ }^{35}$, S.Haug ${ }^{33}$, F.Hauler ${ }^{19}$, V.Hedberg ${ }^{9,25}$, S.Heising ${ }^{19}$, J.J.Hernandez ${ }^{50}$,

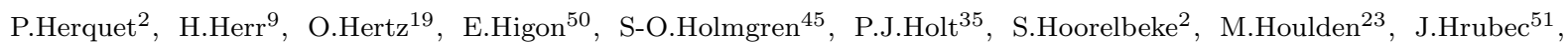
G.J.Hughes $^{23}$, K.Hultqvist ${ }^{9,45}$, J.N.Jackson ${ }^{23}$, R.Jacobsson ${ }^{9}$, P.Jalocha ${ }^{20}$, Ch.Jarlskog ${ }^{25}$, G.Jarlskog ${ }^{25}$, P.Jarry ${ }^{40}$, B.Jean-Marie ${ }^{21}$, D.Jeans ${ }^{35}$, E.K.Johansson ${ }^{45}$, P.Jonsson ${ }^{26}$, C.Joram ${ }^{9}$, P.Juillot ${ }^{10}$, L.Jungermann ${ }^{19}$, F.Kapusta ${ }^{24}$, K.Karafasoulis ${ }^{12}$, S.Katsanevas ${ }^{26}$, E.C.Katsoufis ${ }^{32}$, R.Keranen ${ }^{19}$, G.Kernel ${ }^{44}$, B.P.Kersevan ${ }^{44}$, Yu.Khokhlov ${ }^{43}$, B.A.Khomenko ${ }^{18}$, N.N.Khovanski ${ }^{18}$, A.Kiiskinen ${ }^{17}$, B.King ${ }^{23}, \quad$ A.Kinvig ${ }^{23}$, N.J.Kjaer ${ }^{9}, \quad$ O.Klapp ${ }^{53}, \quad$ P.Kluit ${ }^{31}$, P.Kokkinias ${ }^{12}$, V.Kostioukhine ${ }^{43}$, C.Kourkoumelis ${ }^{3}$, O.Kouznetsov ${ }^{18}$, M.Krammer ${ }^{51}$, E.Kriznic ${ }^{44}$, Z.Krumstein ${ }^{18}$, P.Kubinec $^{7}$, M.Kucharczyk ${ }^{20}$, J.Kurowska ${ }^{52}$, J.W.Lamsa ${ }^{1}$, J-P.Laugier ${ }^{40}$, G.Leder ${ }^{51}$, F.Ledroit ${ }^{16}$, L.Leinonen ${ }^{45}$, A.Leisos ${ }^{12}$, R.Leitner ${ }^{30}$, J.Lemonne ${ }^{2}$, G.Lenzen ${ }^{53}$, V.Lepeltier ${ }^{21}$, T.Lesiak ${ }^{20}$, M.Lethuillier ${ }^{26}$, J.Libby ${ }^{35}$, W.Liebig ${ }^{53}$, D.Liko ${ }^{9}$, A.Lipniacka ${ }^{45}$, I.Lippi ${ }^{36}$, J.G.Loken ${ }^{35}$, J.H.Lopes ${ }^{48}$, J.M.Lopez ${ }^{41}$, R.Lopez-Fernandez ${ }^{16}$, D.Loukas ${ }^{12}$, P.Lutz ${ }^{40}$, L.Lyons $^{35}$, J.MacNaughton ${ }^{51}$, J.R.Mahon ${ }^{6}$, A.Maio ${ }^{22}$, A.Malek ${ }^{53}$, S.Maltezos ${ }^{32}$, V.Malychev ${ }^{18}$, F.Mandl ${ }^{51}$, J.Marco ${ }^{41}$, R.Marco $^{41}$, B.Marechal ${ }^{48}$, M.Margoni ${ }^{36}$, J-C.Marin ${ }^{9}$, C.Mariotti ${ }^{9}$, A.Markou ${ }^{12}$, C.Martinez-Rivero ${ }^{9}$, S.Marti i Garcia ${ }^{9}$, J.Masik $^{13}$, N.Mastroyiannopoulos ${ }^{12}$, F.Matorras ${ }^{41}$, C.Matteuzzi ${ }^{29}$, G.Matthiae ${ }^{38}$, F.Mazzucato ${ }^{36,14}$, M.Mazzucato ${ }^{36}$, M.Mc Cubbin ${ }^{23}$, R.Mc Kay ${ }^{1}$, R.Mc Nulty ${ }^{23}$, G.Mc Pherson ${ }^{23}$, E.Merle ${ }^{16}$, C.Meroni ${ }^{28}$, W.T.Meyer ${ }^{1}$, A.Miagkov ${ }^{43}$, E.Migliore $^{9}$, L.Mirabito ${ }^{26}$, W.A.Mitaroff ${ }^{51}$, U.Mjoernmark ${ }^{25}$, T.Moa ${ }^{45}$, M.Moch ${ }^{19}$, K.Moenig ${ }^{9,11}$, M.R.Monge ${ }^{15}$, J.Montenegro $^{31}$, D.Moraes ${ }^{48}$, P.Morettini ${ }^{15}$, G.Morton ${ }^{35}$, U.Mueller ${ }^{53}$, K.Muenich ${ }^{53}$, M.Mulders ${ }^{31}$, L.M.Mundim ${ }^{6}$, W.J.Murray ${ }^{37}$, B.Muryn ${ }^{20}$, G.Myatt ${ }^{35}$, T.Myklebust ${ }^{33}$, M.Nassiakou ${ }^{12}$, F.L.Navarria ${ }^{5}$, K.Nawrocki ${ }^{52}$, P.Negri ${ }^{29}$, S.Nemecek $^{13}$, N.Neufeld ${ }^{51}$, R.Nicolaidou ${ }^{40}$, P.Niezurawski ${ }^{52}$, M.Nikolenko ${ }^{10,18}$, V.Nomokonov ${ }^{17}, \quad$ A.Nygren ${ }^{25}$, V.Obraztsov $^{43}$, A.G.Olshevski ${ }^{18}$, A.Onofre ${ }^{22}$, R.Orava ${ }^{17}$, K.Osterberg ${ }^{9}$, A.Ouraou ${ }^{40}$, A.Oyanguren ${ }^{50}$, M.Paganoni $^{29}$, S.Paiano $^{5}$, R.Pain ${ }^{24}$, R.Paiva ${ }^{22}$, J.Palacios ${ }^{35}$, H.Palka ${ }^{20}$, Th.D.Papadopoulou ${ }^{32}$, L.Pape ${ }^{9}$, C.Parkes ${ }^{9}$, F.Parodi ${ }^{15}$, U.Parzefall $^{23}$, A.Passeri ${ }^{39}$, O.Passon ${ }^{53}$, L.Peralta ${ }^{22}$, V.Perepelitsa ${ }^{50}$, M.Pernicka ${ }^{51}$, A.Perrotta ${ }^{5}$, C.Petridou ${ }^{47}$, A.Petrolini ${ }^{15}$, H.T.Phillips ${ }^{37}$, F.Pierre ${ }^{40}$, M.Pimenta ${ }^{22}$, E.Piotto ${ }^{28}$, T.Podobnik ${ }^{44}$, V.Poireau ${ }^{40}$, M.E.Pol ${ }^{6}$, G.Polok ${ }^{20}$, P.Poropat $^{47}$, V.Pozdniakov ${ }^{18}$, P.Privitera ${ }^{38}$, N.Pukhaeva ${ }^{18}$, A.Pullia ${ }^{29}$, D.Radojicic ${ }^{35}$, S.Ragazzi ${ }^{29}$, H.Rahmani ${ }^{32}$, A.L.Read ${ }^{33}$, P.Rebecchi ${ }^{9}$, N.G.Redaelli ${ }^{29}$, M.Regler ${ }^{51}$, J.Rehn ${ }^{19}$, D.Reid ${ }^{31}$, R.Reinhardt ${ }^{53}$, P.B.Renton ${ }^{35}$, L.K.Resvanis $^{3}$, F.Richard ${ }^{21}$, J.Ridky ${ }^{13}$, G.Rinaudo ${ }^{46}$, I.Ripp-Baudot ${ }^{10}$, A.Romero ${ }^{46}$, P.Ronchese ${ }^{36}$, E.I.Rosenberg ${ }^{1}$, P.Rosinsky ${ }^{7}$, T.Rovelli ${ }^{5}$, V.Ruhlmann-Kleider ${ }^{40}$, A.Ruiz ${ }^{41}$, H.Saarikko ${ }^{17}$, Y.Sacquin ${ }^{40}$, A.Sadovsky ${ }^{18}$, G.Sajot ${ }^{16}$, L.Salmi ${ }^{17}$, J.Salt ${ }^{50}$, D.Sampsonidis ${ }^{12}$, M.Sannino ${ }^{15}$, A.Savoy-Navarro ${ }^{24}$, C.Schwanda ${ }^{51}$, Ph.Schwemling ${ }^{24}$, B.Schwering ${ }^{53}$, U.Schwickerath ${ }^{19}$, F.Scuri ${ }^{47}$, Y.Sedykh ${ }^{18}$, A.M.Segar ${ }^{35}$, R.Sekulin ${ }^{37}$, G.Sette ${ }^{15}$, R.C.Shellard ${ }^{6}$, M.Siebel ${ }^{53}$, L.Simard ${ }^{40}$, F.Simonetto ${ }^{36}$, A.N.Sisakian ${ }^{18}$, G.Smadja ${ }^{26}$, N.Smirnov ${ }^{43}$, O.Smirnova ${ }^{25}$, G.R.Smith ${ }^{37}$, O.Solovianov ${ }^{43}$, A.Sopczak ${ }^{19}$, R.Sosnowski ${ }^{52}$, T.Spassov ${ }^{9}$, E.Spiriti ${ }^{39}$, S.Squarcia ${ }^{15}$, C.Stanescu ${ }^{39}$, M.Stanitzki ${ }^{19}$, K.Stevenson ${ }^{35}$, A.Stocchi ${ }^{21}$, J.Strauss ${ }^{51}$, R.Strub ${ }^{10}$, B.Stugu ${ }^{4}$, M.Szczekowski $^{52}$, M.Szeptycka ${ }^{52}$, T.Tabarelli ${ }^{29}$, A.Taffard ${ }^{23}$, F.Tegenfeldt $^{49}$, F.Terranova $^{29}$, J.Timmermans $^{31}$, N.Tinti ${ }^{5}$, L.G.Tkatchev ${ }^{18}$, M.Tobin ${ }^{23}$, S.Todorova ${ }^{9}$, B.Tome ${ }^{22}$, A.Tonazzo ${ }^{9}$, L.Tortora ${ }^{39}$, P.Tortosa ${ }^{50}$, D.Treille ${ }^{9}$, G.Tristram ${ }^{8}$, M.Trochimczuk ${ }^{52}$, C.Troncon ${ }^{28}$, M-L.Turluer ${ }^{40}$, I.A.Tyapkin ${ }^{18}$, P.Tyapkin ${ }^{25}$, S.Tzamarias ${ }^{12}$, O.Ullaland ${ }^{9}$, V.Uvarov ${ }^{43}$, G.Valenti ${ }^{9,5}$, E.Vallazza ${ }^{47}$, P.Van Dam ${ }^{31}$, W.Van den Boeck ${ }^{2}$, J.Van Eldik ${ }^{9,31}$, A.Van Lysebetten ${ }^{2}$, N.van Remortel ${ }^{2}$, I.Van Vulpen ${ }^{31}$, G.Vegni ${ }^{28}$, L.Ventura ${ }^{36}$, W.Venus ${ }^{37,9}$, F.Verbeure ${ }^{2}$, P.Verdier ${ }^{26}$, M.Verlato $^{36}$, L.S.Vertogradov ${ }^{18}$, V.Verzi ${ }^{28}$, D.Vilanova ${ }^{40}$, L.Vitale ${ }^{47}$, E.Vlasov ${ }^{43}$, A.S.Vodopyanov ${ }^{18}$, G.Voulgaris ${ }^{3}$, V.Vrba ${ }^{13}$, 
H.Wahlen ${ }^{53}$, A.J.Washbrook ${ }^{23}$, C.Weiser ${ }^{9}$, D.Wicke ${ }^{9}$, J.H.Wickens ${ }^{2}$, G.R.Wilkinson ${ }^{35}$, M.Winter ${ }^{10}$, M.Witek ${ }^{20}$, G.Wolf $^{9}$, J.Yi ${ }^{1}$, O.Yushchenko ${ }^{43}$, A.Zalewska ${ }^{20}$, P.Zalewski ${ }^{52}$, D.Zavrtanik ${ }^{44}$, E.Zevgolatakos ${ }^{12}$, N.I.Zimin ${ }^{18,25}$, A.Zintchenko ${ }^{18}$, Ph.Zoller ${ }^{10}$, G.Zumerle ${ }^{36}$, M.Zupan $^{12}$

\footnotetext{
${ }^{1}$ Department of Physics and Astronomy, Iowa State University, Ames IA 50011-3160, USA

${ }^{2}$ Physics Department, Univ. Instelling Antwerpen, Universiteitsplein 1, B-2610 Antwerpen, Belgium and IIHE, ULB-VUB, Pleinlaan 2, B-1050 Brussels, Belgium and Faculté des Sciences, Univ. de l'Etat Mons, Av. Maistriau 19, B-7000 Mons, Belgium

${ }^{3}$ Physics Laboratory, University of Athens, Solonos Str. 104, GR-10680 Athens, Greece ${ }^{4}$ Department of Physics, University of Bergen, Allégaten 55, NO-5007 Bergen, Norway

${ }^{5}$ Dipartimento di Fisica, Università di Bologna and INFN, Via Irnerio 46, IT-40126 Bologna, Italy

${ }^{6}$ Centro Brasileiro de Pesquisas Físicas, rua Xavier Sigaud 150, BR-22290 Rio de Janeiro, Brazil and Depto. de Física, Pont. Univ. Católica, C.P. 38071 BR-22453 Rio de Janeiro, Brazil and Inst. de Física, Univ. Estadual do Rio de Janeiro, rua São Francisco Xavier 524, Rio de Janeiro, Brazil ${ }^{7}$ Comenius University, Faculty of Mathematics and Physics, Mlynska Dolina, SK-84215 Bratislava, Slovakia ${ }^{8}$ Collège de France, Lab. de Physique Corpusculaire, IN2P3-CNRS, FR-75231 Paris Cedex 05, France

${ }^{9} \mathrm{CERN}, \mathrm{CH}-1211$ Geneva 23, Switzerland

${ }^{10}$ Institut de Recherches Subatomiques, IN2P3 - CNRS/ULP - BP20, FR-67037 Strasbourg Cedex, France

${ }^{11}$ Now at DESY-Zeuthen, Platanenallee 6, D-15735 Zeuthen, Germany

${ }^{12}$ Institute of Nuclear Physics, N.C.S.R. Demokritos, P.O. Box 60228, GR-15310 Athens, Greece

${ }^{13}$ FZU, Inst. of Phys. of the C.A.S. High Energy Physics Division, Na Slovance 2, CZ-180 40, Praha 8, Czech Republic

${ }^{14}$ Currently at DPNC, University of Geneva, Quai Ernest-Ansermet 24, CH-1211, Geneva, Switzerland

${ }^{15}$ Dipartimento di Fisica, Università di Genova and INFN, Via Dodecaneso 33, IT-16146 Genova, Italy

${ }^{16}$ Institut des Sciences Nucléaires, IN2P3-CNRS, Université de Grenoble 1, FR-38026 Grenoble Cedex, France

${ }^{17}$ Helsinki Institute of Physics, HIP, P.O. Box 9, FI-00014 Helsinki, Finland

${ }^{18}$ Joint Institute for Nuclear Research, Dubna, Head Post Office, P.O. Box 79, RU-101 000 Moscow, Russian Federation

${ }^{19}$ Institut für Experimentelle Kernphysik, Universität Karlsruhe, Postfach 6980, DE-76128 Karlsruhe, Germany

${ }^{20}$ Institute of Nuclear Physics and University of Mining and Metalurgy, Ul. Kawiory 26a, PL-30055 Krakow, Poland

${ }^{21}$ Université de Paris-Sud, Lab. de l'Accélérateur Linéaire, IN2P3-CNRS, Bât. 200, FR-91405 Orsay Cedex, France

${ }^{22}$ LIP, IST, FCUL - Av. Elias Garcia, 14-1 ${ }^{\circ}$, PT-1000 Lisboa Codex, Portugal

${ }^{23}$ Department of Physics, University of Liverpool, P.O. Box 147, Liverpool L69 3BX, UK

${ }^{24}$ LPNHE, IN2P3-CNRS, Univ. Paris VI et VII, Tour 33 (RdC), 4 place Jussieu, FR-75252 Paris Cedex 05, France

${ }^{25}$ Department of Physics, University of Lund, Sölvegatan 14, SE-223 63 Lund, Sweden

${ }^{26}$ Université Claude Bernard de Lyon, IPNL, IN2P3-CNRS, FR-69622 Villeurbanne Cedex, France

${ }^{27}$ Univ. d'Aix - Marseille II - CPP, IN2P3-CNRS, FR-13288 Marseille Cedex 09, France

${ }^{28}$ Dipartimento di Fisica, Università di Milano and INFN-MILANO, Via Celoria 16, IT-20133 Milan, Italy

${ }^{29}$ Dipartimento di Fisica, Univ. di Milano-Bicocca and INFN-MILANO, Piazza delle Scienze 3, IT-20126 Milan, Italy

${ }^{30}$ IPNP of MFF, Charles Univ., Areal MFF, V Holesovickach 2, CZ-180 00, Praha 8, Czech Republic

${ }^{31}$ NIKHEF, Postbus 41882, NL-1009 DB Amsterdam, The Netherlands

${ }^{32}$ National Technical University, Physics Department, Zografou Campus, GR-15773 Athens, Greece

${ }^{33}$ Physics Department, University of Oslo, Blindern, NO-1000 Oslo 3, Norway

${ }^{34}$ Dpto. Fisica, Univ. Oviedo, Avda. Calvo Sotelo s/n, ES-33007 Oviedo, Spain

${ }^{35}$ Department of Physics, University of Oxford, Keble Road, Oxford OX1 3RH, UK

${ }^{36}$ Dipartimento di Fisica, Università di Padova and INFN, Via Marzolo 8, IT-35131 Padua, Italy

${ }^{37}$ Rutherford Appleton Laboratory, Chilton, Didcot OX11 OQX, UK

${ }^{38}$ Dipartimento di Fisica, Università di Roma II and INFN, Tor Vergata, IT-00173 Rome, Italy

${ }^{39}$ Dipartimento di Fisica, Università di Roma III and INFN, Via della Vasca Navale 84, IT-00146 Rome, Italy

${ }^{40}$ DAPNIA/Service de Physique des Particules, CEA-Saclay, FR-91191 Gif-sur-Yvette Cedex, France

${ }^{41}$ Instituto de Fisica de Cantabria (CSIC-UC), Avda. los Castros s/n, ES-39006 Santander, Spain

${ }^{42}$ Dipartimento di Fisica, Università degli Studi di Roma La Sapienza, Piazzale Aldo Moro 2, IT-00185 Rome, Italy

${ }^{43}$ Inst. for High Energy Physics, Serpukov P.O. Box 35, Protvino, (Moscow Region), Russian Federation

${ }^{44}$ J. Stefan Institute, Jamova 39, SI-1000 Ljubljana, Slovenia and Laboratory for Astroparticle Physics, Nova Gorica Polytechnic, Kostanjeviska 16a, SI-5000 Nova Gorica, Slovenia, and Department of Physics, University of Ljubljana, SI-1000 Ljubljana, Slovenia

${ }^{45}$ Fysikum, Stockholm University, Box 6730, SE-113 85 Stockholm, Sweden

${ }^{46}$ Dipartimento di Fisica Sperimentale, Università di Torino and INFN, Via P. Giuria 1, IT-10125 Turin, Italy

${ }^{47}$ Dipartimento di Fisica, Università di Trieste and INFN, Via A. Valerio 2, IT-34127 Trieste, Italy and Istituto di Fisica, Università di Udine, IT-33100 Udine, Italy

${ }^{48}$ Univ. Federal do Rio de Janeiro, C.P. 68528 Cidade Univ., Ilha do Fundão BR-21945-970 Rio de Janeiro, Brazil

${ }^{49}$ Department of Radiation Sciences, University of Uppsala, P.O. Box 535, SE-751 21 Uppsala, Sweden

${ }^{50}$ IFIC, Valencia-CSIC, and D.F.A.M.N., U. de Valencia, Avda. Dr. Moliner 50, ES-46100 Burjassot (Valencia), Spain

${ }^{51}$ Institut für Hochenergiephysik, Österr. Akad. d. Wissensch., Nikolsdorfergasse 18, AT-1050 Vienna, Austria

${ }^{52}$ Inst. Nuclear Studies and University of Warsaw, Ul. Hoza 69, PL-00681 Warsaw, Poland

${ }^{53}$ Fachbereich Physik, University of Wuppertal, Postfach 100 127, DE-42097 Wuppertal, Germany
} 


\section{Introduction}

The spontaneous symmetry breaking mechanism is a fundamental component of the Standard Model (SM) but no direct experimental evidence for the Higgs particles has been presented so far. Many of the proposed extensions of the Standard Model change the properties of the Higgs particles, either by the effect of new interactions at higher energy scales or directly by assuming a non-minimal Higgs sector. The introduction of a second Higgs doublet is a natural assumption and it can lead to a scenario where a light Higgs particle with suppressed couplings to fermions arises [1].

In the Two Higgs Doublets Models (2HDM), the lightest scalar Higgs boson $\left(h^{0}\right)$ can be produced at LEP either in association with a CP-odd Higgs particle or in association with a $Z^{0}$ boson. The decay branching ratios for the lightest scalar Higgs change with respect to Standard Model ones and its decay to a pair of photons becomes dominant in large regions of the parameter space, while in the Standard Model this branching ratio is $\leq 10^{-3}$. Events with isolated photons in the final state constitute rather distinctive signatures of this fermiophobic scenario.

We present analyses of final states with isolated photons using the data collected by DELPHI at centre-of-mass energies ranging between $189 \mathrm{GeV}$ and $202 \mathrm{GeV}$, corresponding to a total integrated luminosity of about $380 \mathrm{pb}^{-1}$. In this paper we include also the results from an analysis of 6 -jet events relevant to the $2 \mathrm{HDM}$ scenario. The $h^{0} Z^{0}$ production with $h^{0} \rightarrow \gamma \gamma$ has been investigated previously and interpreted in other frameworks: an analysis of previous DELPHI data can be found in reference [2] and results from other LEP experiments can be found in [3]. Results obtained at LEP 1 will be discussed in section 4.

\section{2HDM: the fermiophobic scenario}

The Two Higgs Doublets Models (2HDM) without explicit CP violation [1] are characterised by five physical Higgs bosons: two neutral CP-even bosons $\left(h^{0}, H^{0}\right)$, two charged bosons $\left(H^{ \pm}\right)$, and one neutral CP-odd boson $\left(A^{0}\right)$. The important parameters for describing the 2HDM are the angles $\alpha$ and $\beta$, where $\alpha$ is the mixing angle in the neutral CP-even Higgs sector and $\tan \beta$ is the ratio of the vacuum expectation values of the two Higgs doublets. A seventh parameter is fixed in the symmetry breaking, and is related to the masses of the vector bosons $Z^{0}$ and $W^{ \pm}$which are nowadays extremely well measured $[4]$.

In the framework of $2 \mathrm{HDM}$ there are four different ways in which the Higgs doublets can couple to fermions [5]. The most common choice is the structure assumed in the Minimal Supersymmetric extension to the Standard Model (MSSM) [6] : one of the Higgs doublets couples both to up type quarks and to leptons, and the other doublet couples to down type quarks.

In this paper a model is explored where only one of the Higgs doublets is allowed to couple to fermions (model type I) [1]. The coupling of the lightest CP-even boson, $h^{0}$, to a fermion pair is then proportional to $\cos \alpha$. If $\alpha=\frac{\pi}{2}$ this coupling vanishes and $h^{0}$ becomes a fermiophobic Higgs.

In general $2 \mathrm{HDM}$, the main mechanisms for the production of neutral Higgs bosons at LEP are $e^{+} e^{-} \rightarrow h^{0} Z^{0}$ and $e^{+} e^{-} \rightarrow h^{0} A^{0}$. These processes have complementary crosssections, proportional to $\sin ^{2} \delta$ and to $\cos ^{2} \delta$ respectively, where $\delta=\alpha-\beta$. The high $\delta$ region can be studied by analysing the Higgs-strahlung process, while the small $\delta$ region is dominated by the associated $h^{0} A^{0}$ production. The combination of both processes 
leads to an interpretation of the results as a function of $m_{h^{0}}$ and $m_{A^{0}}$. The region in the plane $\left(m_{h^{0}}, m_{A^{0}}\right)$ that is relevant for the present analyses corresponds to a band $m_{\text {low }}<m_{A^{0}}+m_{h^{0}}<m_{\text {high }}$. The upper constraint represents the sensitivity accessible with the present LEP 2 integrated luminosity and centre-of-mass energies and the lower constraint corresponds to the region excluded by previous analyses, namely at LEP 1 .

The Higgs-Higgs interactions, namely the $h^{0} H^{+} H^{-}$vertex, depend on the specific 2HDM potential. In fact, there are two different potentials, defined by seven parameters, which assure no $\mathrm{CP}$ violation. They are referred to as potential $\mathrm{A}$ and potential $\mathrm{B}$ [1]. These potentials are equivalent so far as the Higgs couplings to gauge bosons and fermions are concerned. However, differences in the Higgs-Higgs interactions lead to different phenomenologies and can alter the decay width of $h^{0} \rightarrow \gamma \gamma$, for which the $H^{+}$ loop has a fundamental contribution. On the other hand, the relevant tree-level decays of $A^{0}$ are completely independent of the chosen potential. The two potentials also give rise to different forbidden regions in the parameter space accessible at LEP. Namely, a small value of $\delta$ implies a light $h^{0}$ for potential A and a small difference between $m_{h^{0}}$ and $m_{A^{0}}$ for potential B (which is also the one assumed in the MSSM).

In this paper the results are interpreted for both potentials. For Potential A, the branching ratio of the lightest scalar Higgs $\left(h^{0}\right)$ to two photons, $\operatorname{BR}\left(h^{0} \rightarrow \gamma \gamma\right)$, depends only on $m_{h^{0}}$ and mildly on the value of $\delta$, provided that $m_{H^{ \pm}}$is above the experimental limit of $78.6 \mathrm{GeV} / c^{2}[7]$ and the heavier neutral scalar Higgs boson $\left(H^{0}\right)$ has a mass of the order of $1 \mathrm{TeV} / \mathrm{c}^{2}$. For potential $\mathrm{B}$, the same branching ratio depends also on $m_{A^{0}}$ and $m_{H^{ \pm}}$, and there can be large cancellations between the several loop contributions for some values of these parameters. For higher values of $m_{H^{ \pm}}$(above $400 \mathrm{GeV} / c^{2}$ ) or higher values of $\delta\left(\sin ^{2} \delta>0.02\right)$, there are again regions free of such cancellations.

The dominant decay modes for $m_{h^{0}}<m_{Z^{0}}$ in the fermiophobic limit (Model I and $\alpha=\frac{\pi}{2}$ ) are $h^{0} \rightarrow A^{0} A^{0}$ (tree level) if $m_{h^{0}}>2 m_{A^{0}}$ and $h^{0} \rightarrow \gamma \gamma$ (one-loop) otherwise. The decays of $h^{0}$ to other boson pairs can be important when $m_{h^{0}}>m_{Z^{0}}$, namely the one loop decay $h^{0} \rightarrow Z^{0} \gamma$ can have a BR as large as $20 \%$ for very small $\delta$ values, while the decay to $W W^{*}$ is important for large $\delta$ values.

The tree level decay modes of the $A^{0}$ boson are: $A^{0} \rightarrow f \bar{f}, A^{0} \rightarrow Z^{0} h^{0}$, and $A^{0} \rightarrow$ $W^{ \pm} H^{ \pm}$(when kinematicaly allowed). The main decay of $A^{0}$ is into a fermion-antifermion pair, namely a $b \bar{b}$ pair if $m_{A^{0}}>10 \mathrm{GeV}$. However, above the $Z^{0} h^{0}$ threshold, the decay $A^{0} \rightarrow Z^{0} h^{0}$ dominates for all $\delta<1.3 \mathrm{rad}$. Finally it should be noted that in the region of very low $\delta$ values $\left(\delta<10^{-3} \mathrm{rad}\right)$ and $m_{A^{0}}<m_{Z^{0}}+m_{h^{0}}$, the $A^{0}$ total width is very small and $A^{0}$ can leave the detector before decaying [1]. While for potential $\mathrm{B}$, final states with invisible $A^{0}$ are important only for a small band of $m_{A^{0}} \sim m_{h^{0}}$, for potential A they can give rise to totally invisible final states.

The several topologies contributing to the analyses are summarised in table 1 . For $m_{h^{0}}>2 m_{A^{0}}$, the final states will not involve photons but rather 6 b-jets or only invisible particles (stable $A^{0}$ ). In this region the analysis of [8] was used together with the interpretation of LEP 1 data.

\section{Data samples, event selection and analysis}

The analysed data from the LEP runs of 1998 and 1999 were taken at centre-of-mass energies of $189 \mathrm{GeV}, 192 \mathrm{GeV}, 196 \mathrm{GeV}, 200 \mathrm{GeV}$ and $202 \mathrm{GeV}$, with integrated luminosities of about $153,26,77,85$ and $42 \mathrm{pb}^{-1}$, respectively. A detailed description of the DELPHI detector and its performance can be found in references $[9,10]$. The most relevant subdetectors for the present analyses were the electromagnetic calorimeters: the 


\begin{tabular}{|l|c|c|}
\hline Process & Final states & Relevant mass region \\
\hline \hline $\mathrm{e}^{+} \mathrm{e}^{-} \rightarrow \mathrm{h}^{0} \mathrm{~A}^{0}$ & $\gamma \gamma A^{0}$ (long lived) & $\mathrm{m}_{\mathrm{A}^{0}}<\mathrm{m}_{\mathrm{Z}^{0}}+\mathrm{m}_{\mathrm{h}^{0}}$ \\
& $\gamma \gamma \mathrm{b} \overline{\mathrm{b}}$ & $\mathrm{m}_{\mathrm{h}^{0}}+\mathrm{m}_{\mathrm{A}^{0}}>10 \mathrm{GeV} / \mathrm{c}^{2}$ \\
\hline $\mathrm{e}^{+} \mathrm{e}^{-} \rightarrow \mathrm{h}^{0} \mathrm{~A}^{0} \rightarrow \mathrm{h}^{0} \mathrm{~h}^{0} \mathrm{Z}^{0}$ & $\gamma \gamma \gamma \gamma \nu \bar{\nu}$ & $\mathrm{m}_{\mathrm{A}^{0}}>\mathrm{m}_{\mathrm{Z}^{0}}+\mathrm{m}_{\mathrm{h}^{0}}$ \\
& $\gamma \gamma \gamma \gamma \mathrm{q} \overline{\mathrm{q}}$ & \\
\hline $\mathrm{e}^{+} \mathrm{e}^{-} \rightarrow \mathrm{h}^{0} \mathrm{Z}^{0}$ & $\gamma \gamma \nu \bar{\nu}$ & $\mathrm{m}_{\mathrm{h}^{0}}<110 \mathrm{GeV} / \mathrm{c}^{2}$ \\
& $\gamma \gamma \mathrm{q} \overline{\mathrm{q}}$ & \\
\hline
\end{tabular}

Table 1: Topologies of the final states considered in the framework of the explored fermiophobic scenario in 2HDM.

High density Projection Chamber (HPC) in the barrel region, the Forward ElectroMagnetic Calorimeter (FEMC) in the endcaps and the Small angle TIle Calorimeter (STIC) for the very-forward region; the Hadronic CALorimeter (HCAL, covering polar angles down to 11 degrees), and the tracking devices, namely: the Vertex Detector (VD), the Inner Detector (ID), the Time Projection Chamber (TPC) and the Outer Detector (OD) in the barrel and the Forward Chambers A and B (FCA, FCB) in the forward region. The Vertex Detector is crucial for the determination of secondary vertices and the tagging of $b$-quark jets and also for the identification of photons which convert inside the tracking system but after the VD.

The effects of experimental resolution on background and signal events were studied by generating Monte Carlo events and passing them through the full DELPHI simulation and reconstruction chain [10]. The PYTHIA [11] generator was used to simulate the background processes: $\mathrm{e}^{+} \mathrm{e}^{-} \rightarrow \mathrm{Z}^{0}(\mathrm{~N} \gamma) \rightarrow \mathrm{q} \overline{\mathrm{q}}(\mathrm{N} \gamma), \mathrm{e}^{+} \mathrm{e}^{-} \rightarrow \mathrm{W}^{+} \mathrm{W}^{-}$, $\mathrm{e}^{+} \mathrm{e}^{-} \rightarrow \mathrm{W}^{ \pm} \mathrm{e}^{\mp} \nu, \mathrm{e}^{+} \mathrm{e}^{-} \rightarrow \mathrm{Z}^{0} \mathrm{Z}^{0} / \gamma^{*}$, and $\mathrm{e}^{+} \mathrm{e}^{-} \rightarrow \mathrm{Z}^{0} \mathrm{e}^{+} \mathrm{e}^{-}$. The $\mathrm{e}^{+} \mathrm{e}^{-} \rightarrow \mathrm{Z}^{0}(\mathrm{~N} \gamma) \rightarrow \nu \bar{\nu}(\mathrm{N} \gamma)$, $\mathrm{e}^{+} \mathrm{e}^{-} \rightarrow \mathrm{Z}^{0}(\mathrm{~N} \gamma) \rightarrow \mu \bar{\mu}(\mathrm{N} \gamma)$ and $\mathrm{e}^{+} \mathrm{e}^{-} \rightarrow \mathrm{Z}^{0}(\mathrm{~N} \gamma) \rightarrow \tau \bar{\tau}(\mathrm{N} \gamma)$ processes were generated with the KoralZ generator [12]. Bhabha events were generated with the BHWIDE generator [13], $\mathrm{e}^{+} \mathrm{e}^{-} \rightarrow \gamma \gamma(\gamma)$ events according to [14], and Compton events according to [15]. The two-photon (" $\gamma \gamma$ ") physics events were generated with the TWOGAM [16] generator.

The two main backgrounds in the analysis are $\mathrm{e}^{+} \mathrm{e}^{-} \rightarrow \mathrm{q} \overline{\mathrm{q}}(\mathrm{N} \gamma)$ and $\mathrm{e}^{+} \mathrm{e}^{-} \rightarrow \nu \bar{\nu}(\mathrm{N} \gamma)$. The matrix-element in KoralZ generator (used for $\nu \bar{\nu}(N \gamma)$ ) has the complete order $\alpha$ complemented with a third order leading-log expansion. The Pyhia generator (used for $q \bar{q}(N \gamma)$ ) was verified to be compatible with KoralZ for events with up to two visible photons. The absence of a complete description of the multiple photon radiation in $\mathrm{MC}$ generators may be a problem for very high luminosity analysis.

The analysis of events with isolated photons was done in several steps. First a general selection was applied and isolated leptons, isolated photons and jets were reconstructed. Events with isolated leptons were removed from the analysis.

Charged particles were considered only if they had momentum greater than $0.1 \mathrm{GeV} / c$ and impact parameters below $4 \mathrm{~cm}$ in the transverse plane and below $4 \mathrm{~cm} / \sin \theta$ in the beam direction ( $\theta$ is the polar angle, defined in relation to the beam axis). Energy deposits in the calorimeters unassociated to charged particle tracks were required to be above $0.3 \mathrm{GeV}$.

Isolated particles were defined by constructing double cones centered around the axis of the neutral cluster (charged particle track) with half opening angles of $5^{\circ}$ and $15^{\circ}$ $\left(5^{\circ}\right.$ and $\left.25^{\circ}\right)$, and requiring that the average energy density in the outer cone was below $10 \mathrm{MeV} /$ degree ( $15 \mathrm{MeV} /$ degree), to assure isolation. In the case of neutral deposits, 
no charged particle with more than $250 \mathrm{MeV}$ was allowed inside the inner cone. The energy of the isolated particle was then re-evaluated as the sum of the energies (charged particle track momenta) inside the inner cone. For well identified photons or leptons, the above requirements were weakened: the external angle was allowed to be smaller and one energetic particle was allowed in the outer cone.

Photons were further required to have no HPC layer with more than $90 \%$ of the photon electromagnetic energy. Alternatively, energy deposits above $3 \mathrm{GeV}$ in the hadronic calorimeter were considered as photon candidates if at least $90 \%$ of the deposited energy was in the first layer of the HCAL.

Photons converting within the tracking system were recovered only in the non-hadronic topologies.

\subsection{Photonic final states}

Photons converting inside the tracking system, but after the Vertex Detector, are characterized by charged particle tracks and will be referred to as converted photons. Photons reaching the electromagnetic calorimeters before converting, yielding no reconstructed charged particles tracks, will be referred to as unconverted photons. According to this classification, two different algorithms were applied in the photon reconstruction and identification.

Energy deposits were considered unconverted photons if the following requirements were fulfilled:

- The energy was above $3 \mathrm{GeV}$.

- The polar angle of the energy deposit was inside one of the intervals $\left[20^{\circ}, 35^{\circ}\right]$, $\left[42^{\circ}, 88^{\circ}\right],\left[92^{\circ}, 138^{\circ}\right]$ or $\left[145^{\circ}, 160^{\circ}\right]$ in order to reduce calorimeter edge effects.

- No charged particle tracks were associated to the energy deposit.

- There was no VD track element pointing to the energy deposit direction within $3^{\circ}$ $\left(10^{\circ}\right)$ in azimuth in the barrel (forward) region of DELPHI (a VD track element was defined as at least two hits in different VD layers aligned within an azimuthal angle interval of $0.5^{\circ}$, assuming the charged particle track originated from the beam spot).

- If the polar angle of the energy deposit was below $30^{\circ}$ (above $150^{\circ}$ ), it had to be out of the 6 TPC $\phi$ intermodular divisions by $2.5^{\circ}$.

Photons converting after the VD in the polar angle range between $25^{\circ}$ and $155^{\circ}$ were recovered. They were reconstructed with the help of the DURHAM jet clustering algorithm [17]. All particles in the event, with exception of isolated neutral particles were clustered in jets, using as the resolution variable $y_{\text {cut }}=0.003$. Low multiplicity jets with less than 6 charged particles were treated as converted photon candidates if they were associated to energy deposits fulfilling the same requirements imposed on unconverted photons.

A common preselection was defined for all the photonic final states (level 1). It was required that the visible energy in the polar angle region between $20^{\circ}$ and $160^{\circ}$ was greater than $0.1 \sqrt{s}$. The number of charged particle tracks was required to be less than 6 , all without VD track elements. At least two photons had to have energy greater than 5 $\mathrm{GeV}$ and polar angles between $25^{\circ}$ and $155^{\circ}$. No particles (with the exception of isolated photons) with energy above $3 \mathrm{GeV}$ were allowed in the event; no more than one photon converting in the tracking system was allowed.

Specific criteria were then applied to the photonic preselected sample according to the final state topology under study. 


\subsubsection{Events with two photons and missing energy}

The level 2 selection of the $\gamma \gamma+E_{\text {miss }}$ sample consisted of requiring events with two and only two photons. The acoplanarity ${ }^{1}$ between the two photons in these events is compared to the Standard Model prediction in figure 1a).

Final selection criteria (level 3), aiming at the enhancement of a possible signal contribution were then imposed and consisted of the following conditions:

- Whenever the missing momentum was greater than $0.1 \sqrt{s}$ the polar angle of the direction of the missing momentum was required to be greater than $10^{\circ}$ and less than $170^{\circ}$ and no signal in the set of lead/scintillator counters placed between the barrel and forward electromagnetic calorimeters was allowed.

- The acoplanarity between the two photons was required to be greater then $10^{\circ}$.

- The sum of the energies of the two photons had to be lower than $0.7 \sqrt{s}$.

In the case of the search for the Higgs-strahlung production, $h^{0} Z^{0}$, with $h^{0} \rightarrow \gamma \gamma$ and $Z^{0} \rightarrow \nu \bar{\nu}$, it was further required that the mass recoiling against the two photons was above $20 \mathrm{GeV} / c^{2}$. The invariant masses of the photon pairs are displayed for these events in figure $1 b)$. The background comes mainly from double radiative returns to the $Z^{0}$ with $Z \rightarrow \nu \bar{\nu}$.

The efficiencies are about $60 \%$ for both $h^{0} Z^{0}$ and $h^{0} A^{0}$, for all centre-of-mass energies and mass ranges considered. For $m_{h^{0}}=90 \mathrm{GeV} / c^{2}$ and $\delta=\pi / 4$, the number of expected signal events from $h^{0} Z^{0}$ production is 1.7 .

\subsubsection{Events with four photons and missing energy}

Different criteria were imposed on the level 1 photonic sample in order to get a wide sample of candidates for the associated production of $h^{0} A^{0}$, in which the CP-odd boson decays to $h^{0} Z^{0}$, the $Z^{0}$ going to two neutrinos. The specific criteria for selecting $\gamma \gamma \gamma \gamma+E_{\text {miss }}$ events (level 2), consisted of demanding that the events had at least three photons, all but one between $25^{\circ}$ and $155^{\circ}$ in polar angle. Moreover, whenever the missing momentum was greater than $0.1 \sqrt{s}$ the polar angle of the direction of the missing momentum was required to be between $10^{\circ}$ and $170^{\circ}$.

A final set of requirements was imposed in order to enhance a possible signal (selection level 3):

- The acoplanarity between the two most energetic photons had to be greater then $10^{\circ}$.

- If the missing energy was below $70 \mathrm{GeV}$, the missing transverse momentum had to be greater that $50 \mathrm{GeV} / c$.

- The energy of the most energetic photon had to be less than $\sqrt{s} / 2-20 \mathrm{GeV}$.

The average efficiency of this selection is around $50 \%$. For $m_{h^{0}}=10 \mathrm{GeV} / c^{2}$ and $m_{A^{0}}=120 \mathrm{GeV} / c^{2}$ and a $\delta=\pi / 4$, the signal expectation is of 3.6 events, for a total background expectation of $2.9 \pm 0.5$ events, coming both from $Z^{0} \gamma \gamma$ and $\gamma \gamma$ producton.

\subsection{Final states with jets and photons}

Selection criteria were implemented to identify events with two jets and at least two isolated photons (level 1). Isolated photons were reconstructed as explained in the be-

\footnotetext{
${ }^{1}$ acoplanarity is defined as the complement of the angle between the projections of the two photons in the plane perpendicular to the beam
} 
gining of section 3. Their energy was further required to be above $5 \mathrm{GeV}$ to avoid large contamination from photons coming from the hadronization.

Events were selected in the hadronic topologies if at least six charged particles were present, the visible energy in the polar angle region between $20^{\circ}$ and $160^{\circ}$ was greater than $0.2 \sqrt{s}$ and there was at least one charged particle or one electromagnetic cluster with an energy greater than $5 \mathrm{GeV}$. All selected charged particles and neutrals not associated to isolated photons were forced to be clustered into two jets using the DURHAM jet algorithm [17].

For $q \bar{q} \gamma \gamma,(q \bar{q} \gamma \gamma \gamma \gamma)$ final states two (at least three) photons with polar angle above $40^{\circ}$ and below $140^{\circ}$ were required. In order to improve momentum and energy resolution for the $q \bar{q} \gamma \gamma$ final states, a kinematic fit [18] imposing total energy and momentum conservation (with the two jets and two photons) was performed on the selected events. Only events with a $\chi^{2}$ per degree of freedom lower than 5 were accepted. This defined the selection level 2. The jet-jet mass resolution at this level was $3 \mathrm{GeV} / c^{2}$.

Selection level 2 was used for the search for $h^{0} Z^{0}$. A selection level 3 was defined for the search for $h^{0} A^{0}$ with $A^{0} \rightarrow b \bar{b}$, in which flavour tagging was performed based on the identification of the final state quark. Events with a high probability of containing a b quark (using the variable defined in [19]) were thus accepted, allowing for a reduction of $50 \%$ in the background while keeping $90 \%$ of the signal.

The $\gamma \gamma$ invariant masses reconstructed for events with two jets and two photons are displayed in figure 2, both for $h^{0} Z^{0}$ (a) and $h^{0} A^{0}$ (b) searches.

The average efficiencies for masses near the upper kinematic limit are $36 \%$ and $33 \%$ for two photon events from $h^{0} Z^{0}$ and $h^{0} A^{0}$ production, respectively, and $30 \%$ for the final state with at least three photons. These numbers correspond to expectations of 2.4 events from $h^{0} Z^{0}$ in the $q \bar{q} \gamma \gamma$ selection and 2.1 from $h^{0} A^{0}$ in the $b \bar{b} \gamma \gamma$ selection, for $m_{A^{0}}=m_{h^{0}}=90 \mathrm{GeV} / c^{2}$ and $\delta=\pi / 4$. For the final state with at least three photons and for masses of $m_{A^{0}}=120 \mathrm{GeV} / c^{2}$ and $m_{h^{0}}=10 \mathrm{GeV}$ and $\delta=\pi / 4$, the signal expectation is of 5.0 events to be compared with $3.0 \pm 0.6$ background events coming mainly from $q \bar{q} \gamma \gamma \gamma$.

\section{Results}

The number of candidates at different selection levels for the relevant topologies are given in table 2. The numbers in parentheses correspond to the Standard Model expectations which, in the case of final states with only photons, were corrected for trigger efficiencies (of the order of $98 \%$ in the barrel region of the detector and above $99 \%$ in the forward region considered in the analysis). Overall, there is a reasonable agreement between data and $\mathrm{MC}$ expectations.

Small excesses appear in topologies with low statistics. For instance, in the $q \bar{q} \gamma \gamma \gamma \gamma$ final state at $189 \mathrm{GeV}$ there is a slight excess not confirmed at higher energies. The reconstructed $m_{Z^{0}}$ (missing mass or invariant mass of the two jets) for events selected in the last level of the two topologies with four photons are shown in figure 3 . It should be remarked that the good description of the $Z^{0}(N \gamma)$ background has been confirmed only for final states with at most two visible photons.

Signal selection efficiencies were calculated for each final state topology according to the specific process to be studied. Several $\left(m_{h^{0}}, m_{A^{0}}\right)$ points covering the relevant parameter space were considered. For all these masses, the width of the Higgs bosons is smaller than the mass resolution.

These results were then combined and interpreted within the $2 \mathrm{HDM}$ fermiophobic framework giving limits on the cross-sections of the studied processes. The Modified 


\begin{tabular}{|c|c|c|c|c|}
\hline \multirow{2}{*}{$\begin{array}{c}\text { Energy } \\
\text { GeV }\end{array}$} & \multirow{2}{*}{ topology } & \multicolumn{3}{|c|}{ selection level } \\
\hline & & 1 & 2 & 3 \\
\hline \multirow{4}{*}{189} & $\gamma \gamma \gamma \gamma$ & \multirow[b]{2}{*}{$714(707 \pm 6)$} & $91(85 \pm 2)$ & $2(1.5 \pm 0.4)$ \\
\hline & $\gamma \gamma$ & & $561(555 \pm 5)$ & $\begin{array}{lll}7 & (5.6 \pm 0.9) & \left(h^{0} Z^{0}\right) \\
8 & (6.4 \pm 0.9) & \left(h^{0} A^{0}\right)\end{array}$ \\
\hline & $\mathrm{b} \overline{\mathrm{b}} \gamma \gamma$ & $320(359 \pm 7)$ & $26(30 \pm 2)$ & $12(14 \pm 1)$ \\
\hline & $\mathrm{q} \overline{\mathrm{q}} \gamma \gamma \gamma \gamma$ & $25(27 \pm 2)$ & \multicolumn{2}{|c|}{$\begin{array}{ll}5 & (1.7 \pm 0.5) \\
\end{array}$} \\
\hline \multirow{4}{*}{192} & $\gamma \gamma \gamma \gamma$ & \multirow[b]{2}{*}{$91(119 \pm 1)$} & $12(13.6 \pm 0.4)$ & $1(0.15 \pm 0.05)$ \\
\hline & $\gamma \gamma$ & & $77(89 \pm 1)$ & $\begin{array}{lll}1 & (1.0 \pm 0.1) & \left(h^{0} Z^{0}\right) \\
1 & (1.1 \pm 0.1) & \left(h^{0} A^{0}\right)\end{array}$ \\
\hline & $\mathrm{b} \overline{\mathrm{b}} \gamma \gamma$ & $48(63 \pm 2)$ & $2(2.8 \pm 0.4)$ & $0(1.0 \pm 0.2)$ \\
\hline & $\mathrm{q} \overline{\mathrm{q}} \gamma \gamma \gamma \gamma$ & $1(4.7 \pm 0.5)$ & \multicolumn{2}{|c|}{$\begin{array}{ll}0 & (0.11 \pm 0.08) \\
\end{array}$} \\
\hline \multirow{4}{*}{196} & $\gamma \gamma \gamma \gamma \gamma$ & \multirow[b]{2}{*}{$343(347 \pm 3)$} & $46(40 \pm 1)$ & $2(0.4 \pm 0.1)$ \\
\hline & $\gamma \gamma$ & & $264(259 \pm 3)$ & 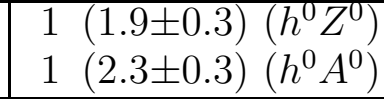 \\
\hline & $\mathrm{b} \overline{\mathrm{b}} \gamma \gamma$ & $165(148 \pm 3)$ & $6(8.6 \pm 0.8)$ & $1(3.6 \pm 0.5)$ \\
\hline & $\mathrm{q} \overline{\mathrm{q}} \gamma \gamma \gamma \gamma$ & $8(10.6 \pm 0.9)$ & \multicolumn{2}{|c|}{$1 \quad(0.7 \pm 0.2)$} \\
\hline \multirow{4}{*}{200} & $\gamma \gamma \gamma \gamma$ & \multirow[b]{2}{*}{$334(356 \pm 3)$} & $43(42 \pm 1)$ & $0(0.6 \pm 0.2)$ \\
\hline & $\gamma \gamma$ & & $263(264 \pm 3)$ & 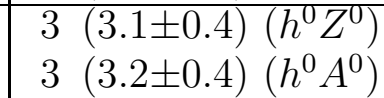 \\
\hline & $\mathrm{b} \overline{\mathrm{b}} \gamma \gamma$ & $149(153 \pm 5)$ & $12(7 \pm 1)$ & $6(2.8 \pm 0.7)$ \\
\hline & $\mathrm{q} \overline{\mathrm{q}} \gamma \gamma \gamma \gamma$ & $13(8 \pm 1)$ & \multicolumn{2}{|c|}{$\begin{array}{rr}2 & (0.4 \pm 0.2) \\
\end{array}$} \\
\hline \multirow{4}{*}{202} & $\gamma \gamma \gamma \gamma$ & \multirow[b]{2}{*}{$171(170 \pm 2)$} & $28(19.8 \pm 0.6)$ & $0(0.29 \pm 0.08)$ \\
\hline & $\gamma \gamma$ & & $126(128 \pm 1)$ & $\begin{array}{lll}2 & (1.2 \pm 0.2) & \left(h^{0} Z^{0}\right) \\
2 & (1.3 \pm 0.2) & \left(h^{0} A^{0}\right) \\
\end{array}$ \\
\hline & $\mathrm{b} \bar{b} \gamma \gamma$ & $82(80 \pm 3)$ & $7(3.8 \pm 0.5)$ & $1(2.1 \pm 0.4)$ \\
\hline & $\mathrm{q} \overline{\mathrm{q}} \gamma \gamma \gamma \gamma$ & $8(5.6 \pm 0.7)$ & 0 & $(0.1 \pm 0.1)$ \\
\hline
\end{tabular}

Table 2: Number of events passing the sets of cuts corresponding to the selection levels described in the text for each topology and centre-of-mass energy. The MC predicted numbers of events and their statistical errors are displayed between parentheses. The second selection level of $b \bar{b} \gamma \gamma$ is the last level for the selection of $q \bar{q} \gamma \gamma$.

Frequentist Likelihood Ratio method described in [20] was used. The method is based on the measured and expected mass distributions. A test statistic is constructed as the ratio of the probability density functions of the signal plus background to background only hypotheses.

\subsection{Constraints from LEP 1}

The production of Higgs bosons at LEP 1 energies would have the effect of increasing the $Z^{0}$ width. Since the $Z^{0}$ parameters are very well measured, tight bounds can be derived on the Higgs mass. However these results should be used with some care [21]. The Higgs production would change the result for the hadronic cross-section, which plays an important role in the fitting of the electroweak parameters. In [21] a fit with more independent variables is performed, assuming that only the $e^{+} e^{-} \rightarrow Z^{0} \rightarrow e^{+} e^{-}$and $e^{+} e^{-} \rightarrow Z^{0} \rightarrow \mu^{+} \mu^{-}$have no contribution from new physics and that the new physics 
corrections to the other processes are not strongly flavour dependent. The limit thus obtained is almost model independent and completely independent of the efficiency with which the new modes could be selected for the $e^{+} e^{-} \rightarrow Z^{0} \rightarrow$ hadrons or $e^{+} e^{-} \rightarrow Z^{0} \rightarrow$ $\tau^{+} \tau^{-}$samples. A 95\% Confidence Level (CL) upper limit of $6.3 \mathrm{MeV} / c^{2}$ is obtained for the change in the total $Z^{0}$ width, which yields a limit of $149.4 \mathrm{pb}$ for the production cross-section of unknown particles.

This cross-section limit can be used to constrain both $h^{0} Z^{0^{*}}$ production if $\sin ^{2} \delta=1$ and $h^{0} A^{0}$ production if $\sin ^{2} \delta=0$. The first case corresponds to the exclusion of $m_{h^{0}}<$ $9 \mathrm{GeV} / c^{2}$ at $95 \% \mathrm{CL}$ and the second to the exclusion of a band of values corresponding approximately to $m_{h^{0}}+m_{A^{0}}<70 \mathrm{GeV} / c^{2}$ at $95 \% \mathrm{CL}$. The intersection of the two regions is excluded for all $\delta$ values.

On the other hand, for processes where all the decay products are invisible, the measurement of the $Z^{0}$ invisible width can be used, leading to tighter limits on their crosssection. LEP 1 data [4] leads to a cross-section upper limit of $67 \mathrm{pb}$ at $95 \% \mathrm{CL}$. This limit allows us to exclude the totally invisible final state arising from $h^{0} A^{0} \rightarrow A^{0} A^{0} A^{0}$ and $A^{0}$ stable (using potential $\mathrm{A}$ in the $2 \mathrm{HDM}$ fermiophobic scenario), excluding a band of values corresponding approximately to $m_{h^{0}}+m_{A^{0}}<80 \mathrm{GeV} / c^{2}$ at $95 \%$ CL.

\subsection{Constraints from 6-fermion final states}

In general 2HDM, the decay $h^{0} \rightarrow A^{0} A^{0}$ is the dominant one when kinematically allowed. This gives rise to final states with 6 -jets: $6 b$-jets for $h^{0} A$ production and $4 b$-jets $+Z^{0}$ for the Higgs-strahlung process.

To cover this kinematic region $\left(m_{h^{0}}>2 m_{A^{0}}\right)$, the results from [8] were used. In this paper, there is no dedicated analysis of 6 -jet events, but it is shown that the analysis of 4-jet events (relevant for the SM and MSSM Higgs searches) has enough sensitivity to exclude this region almost up to the kinematic limit.

A $95 \%$ CL exclusion in the plane $\left(m_{h^{0}}, m_{A^{0}}\right)$, valid for all possible combinations of $\alpha$ and $\beta$ (all values of $\delta$ ), is obtained by combining the numbers of expected events in $h^{0} Z^{0}$ and $h^{0} A^{0}$ channels and minimizing the CL with respect to $\sin \delta$ and $\cos \delta$.

\section{Limits on fermiophobic Higgs boson production}

In figure 4 , the $95 \% \mathrm{CL}$ limits on the production of a resonance $X$ in the process $Z^{0} X \rightarrow Z^{0} \gamma \gamma$ as a function of the $\gamma \gamma$ invariant mass are presented in terms of the product of $B R(X \rightarrow \gamma \gamma)$ and $\xi=\sigma\left(Z^{0} X\right) / \sigma\left(Z^{0} H\right)_{S M}$. Here the analyses of $q \bar{q} \gamma \gamma$ and $\nu \bar{\nu} \gamma \gamma$ were used and the limit is valid for resonances with width smaller than the analyses mass resolution. For a value of $\xi \cdot B R=1$, a limit of $107 \mathrm{GeV} / c^{2}$ is obtained for $m_{h^{0}}$. Also shown is $\operatorname{BR}\left(h^{0} \rightarrow \gamma \gamma\right)$ as a function of $m_{h^{0}}$, obtained for potential A of the 2HDM. This branching fraction is comparable to the one obtained in the SM by setting to zero the value of the couplings of the Higgs boson to fermion pairs.

In the 2HDM scenario, $\xi$ corresponds to $\sin ^{2} \delta$ and the BR (which is a function of $\left.m_{h^{0}}, m_{A^{0}}, \delta\right)$ must be taken into account to determine the excluded $\left(m_{h^{0}}, \sin ^{2} \delta\right)$ region. The result for potential A is shown in figure 5. The lower limit thus obtained for $m_{h^{0}}$ is $96 \mathrm{GeV} / c^{2}$ at $95 \% \mathrm{CL}$, for $\sin ^{2} \delta=1$. For small values of $\sin ^{2} \delta$, the Higgs-strahlung cross-section vanishes but an exclusion region can be obtained from the $h^{0} A^{0}$ associated production. Such 95\% CL exclusion regions are shown for two different $A^{0}$ masses. For $m_{A^{0}}<60 \mathrm{GeV} / c^{2}, m_{h^{0}}<9 \mathrm{GeV} / c^{2}$ is excluded by the $Z^{0}$ width measurements. 
Due to the complementarity of the $h^{0} Z^{0}$ and $h^{0} A^{0}$ cross-sections, regions of the plane $\left(m_{h^{0}}, m_{A^{0}}\right)$ can be excluded at $95 \% \mathrm{CL}$ for all $\delta$ values. Figures $6(\mathrm{a})$ and $6(\mathrm{~b})$ show the excluded regions in this plane in the framework of potentials $\mathrm{A}$ and $\mathrm{B}$, respectively. In both plots, Region I corresponds to the zone where $h^{0} \rightarrow \gamma \gamma$ and $A^{0} \rightarrow b \bar{b}$. In the case of potential A, results of $\gamma \gamma A^{0}$ (stable) must also be taken into account, however, the $\gamma \gamma$ final state gives stronger limits and the unexcluded region is still defined by the $b \bar{b} \gamma \gamma$ search. In Region II, corresponding to $h^{0} \rightarrow \gamma \gamma$ and $A^{0} \rightarrow h^{0} Z^{0}$, both $\gamma \gamma \gamma \gamma$ and $q \bar{q} \gamma \gamma \gamma \gamma$ are considered, together with the Higgs-strahlung process. In Region III, corresponding to $h^{0} \rightarrow A^{0} A^{0}$ and $A^{0} \rightarrow b \bar{b}$ giving rise to 6 -jet final states, the $95 \%$ CL limits on $\left(m_{h^{0}}, m_{A^{0}}\right)$ from [8] are used. In the case of potential $\mathrm{A}$, the $A^{0}$ boson can be stable and the limits from the $Z^{0}$ invisible width provide the most conservative exclusion region. As discussed previously, the measurement of the $Z^{0}$ width at LEP 1 allows the exclusion of a band of values for low masses of both $h^{0}$ and $A^{0}$ which is common for both potentials, this is also indicated in figures $6(\mathrm{a})$ and $6(\mathrm{~b})$.

\section{Conclusions}

DELPHI data corresponding to a total integrated luminosity of $380 \mathrm{pb}^{-1}$, at centreof-mass energies between $189 \mathrm{GeV}$ and $202 \mathrm{GeV}$, have been analysed and a search for a neutral Higgs boson with predominantly non-fermionic couplings was performed. The final states $\gamma \gamma, \gamma \gamma \gamma \gamma, \mathrm{b} \bar{b} \gamma \gamma, \mathrm{q} \overline{\mathrm{q}} \gamma \gamma$ and $\mathrm{q} \overline{\mathrm{q}} \gamma \gamma \gamma \gamma$ were considered. A large region of the parameter space in a $2 \mathrm{HDM}$ fermiophobic scenario was excluded.

\section{Acknowledgements}

We would like to thank L.Brücher and R.Santos for very interesting and long discussions in exploring the 2HDM fermiophobic scenario.

We are greatly indebted to our technical collaborators, to the members of the CERNSL Division for the excellent performance of the LEP collider, and to the funding agencies for their support in building and operating the DELPHI detector.

We acknowledge in particular the support of Austrian Federal Ministry of Education, Science and Culture, GZ 616.364/2-III/2a/98, FNRS-FWO, Flanders Institute to encourage scientific and technological research in the industry (IWT), Belgium,

FINEP, CNPq, CAPES, FUJB and FAPERJ, Brazil,

Czech Ministry of Industry and Trade, GA CR 202/96/0450 and GA AVCR A1010521,

Danish Natural Research Council,

Commission of the European Communities (DG XII),

Direction des Sciences de la Matière, CEA, France,

Bundesministerium für Bildung, Wissenschaft, Forschung und Technologie, Germany,

General Secretariat for Research and Technology, Greece,

National Science Foundation (NWO) and Foundation for Research on Matter (FOM),

The Netherlands,

Norwegian Research Council,

State Committee for Scientific Research, Poland, 2P03B06015, 2P03B11116 and SPUB/P03/DZ3/99,

JNICT-Junta Nacional de Investigação Científica e Tecnológica, Portugal,

Vedecka grantova agentura MS SR, Slovakia, Nr. 95/5195/134,

Ministry of Science and Technology of the Republic of Slovenia, 
CICYT, Spain, AEN96-1661 and AEN96-1681, The Swedish Natural Science Research Council,

Particle Physics and Astronomy Research Council, UK, Department of Energy, USA, DE-FG02-94ER40817. 


\section{References}

[1] A. Barroso, L. Brücher, R. Santos, Phys. Rev. D60 (1999) 035005

L. Brücher, R. Santos, Eur. Phys. J. C12 (2000) 87

[2] DELPHI Coll., P. Abreu et al., Phys. Lett. B458 (1999) 431

[3] ALEPH Coll.,R. Barate et al., CERN-EP/2000-083 (2000), submitted to Phys. Lett. $\mathrm{B}$

L3 Coll., "Search for a Higgs Boson Decaying into Two Photons in $e+e-$ Interactions at centre-of-mass energies up to $202 \mathrm{GeV}$ at LEP", L3 note 2580, contributed paper to ICHEP'2000, Osaka, 2000

OPAL Coll.,G. Abbiendi et al., Phys. Lett. B464 (1999) 311

[4] LEP and SLD Coll., "A combination of Preliminary Electroweak Measurements and Constraints on the Standard Model", CERN-EP-2000-016

[5] A G Akeroyd, Nucl. Phys. B544 (1999) 557

J.F. Gunion, H.E.Haber, G.L.Kane and S. Dawnson, "The Higgs Hunter's Guide" (Addison-Wesley, Reading 1990)

[6] See for example:

H.P.Nilles, Phys. Rep. 110 (1984)1

M.E.Haber and G.L.Kane, Phys. Rep. 117(1985)75

M.F.Sohnius, Phys. Rep. 128(1985)39

[7] ALEPH, DELPHI, L3 and OPAL Coll. and the LEP working group for Higgs boson searches, "Searches for Higgs bosons: Preliminary combined results using LEP data collected at energies up to $202 \mathrm{GeV}$ ", CERN-EP-2000-055

[8] DELPHI Coll., "Search for Neutral Higgs Bosons in $e+e-$ Collisions at $\sqrt{s}=188.7$ GeV", DELPHI 99-88 CONF 173, contributed paper to HEP'99, Tampere, 1999

[9] DELPHI Coll., P. Aarnio et al., Nucl. Instr. and Meth. A303 (1991) 233

[10] DELPHI Coll., P. Abreu et al., Nucl. Instr. and Meth. A378 (1996) 57

[11] T. Sjöstrand, Comp. Phys. Comm. 82 (1994) 74,

T. Sjöstrand, Pythia 5.7 and Jetset 7.4, Cern-TH/7112-93

[12] S. Jadach et al., Comp. Phys. Comm. 66 (1991) 276,

S. Jadach et al., Comp. Phys. Comm. 79 (1994) 503

[13] S. Jadach, W. Placzek and B.F.L. Ward, Phys. Lett. B390 (1997) 298

[14] F. Berends and R. Kleiss, Nucl. Phys. B186 (1981) 22

[15] D. Karlen, Nucl. Phys. B289 (1987) 23

[16] S. Nova, A. Olchevski and T. Todorov, in "Physics at LEP2", ed. G. Altarelli, T. Sjöstrand and F.Zwirner, CERN Report 96-01, Vol.2,(1996) p.224

[17] S. Catani et al., Phys. Lett. B269 (1991) 432

[18] DELPHI Coll., P. Abreu et al., Eur. Phys. J. C2 (1998) 581

[19] G. Borisov, Nucl. Instr. and Meth. A417 (1998) 384

[20] A.L. Read, "Modified Frequentist Analysis of Search Results (The CLs Method)" in "Workshop on Confidence Limits", ed. F. James, L. Lyons and Y.Perrin, CERN Report 2000-005 (2000) p.81

[21] K. Mönig, "Model Independent Limit of the Z-Decay-Width into Unknown Particles", CERN-OPEN-97-040

see also: [4] 

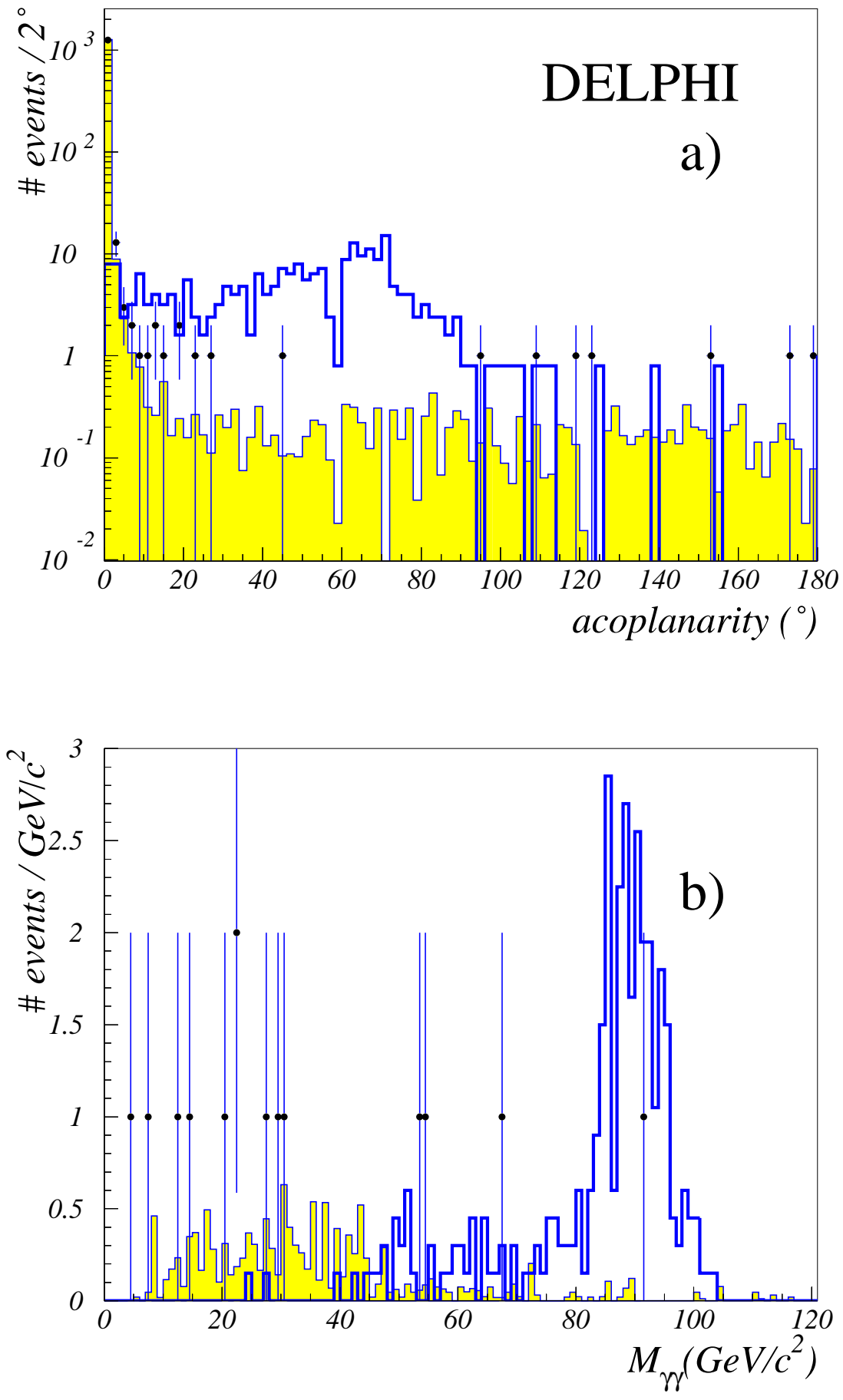

Figure 1: Acoplanarity between the two photons at selection level 2 (a) and invariant $\gamma \gamma$ mass at selection level 3 (b) of the two photon analysis. The dots represent the data. The shaded area represents the expected standard model background, which comes mainly from the process $e^{+} e^{-} \rightarrow Z^{0} \rightarrow \nu \bar{\nu}$ with two visible ISR photons and from the QED process $e^{+} e^{-} \rightarrow \gamma \gamma(\gamma)$. The darker line corresponds to signal distributions for a Higgs mass of $90 \mathrm{GeV} / c^{2}$, with arbitrary normalization. The invariant mass distribution corresponds to level 3 of the $h^{0} Z^{0}$ selection, with 14 data events and $13 \pm 1$ expected from background. 

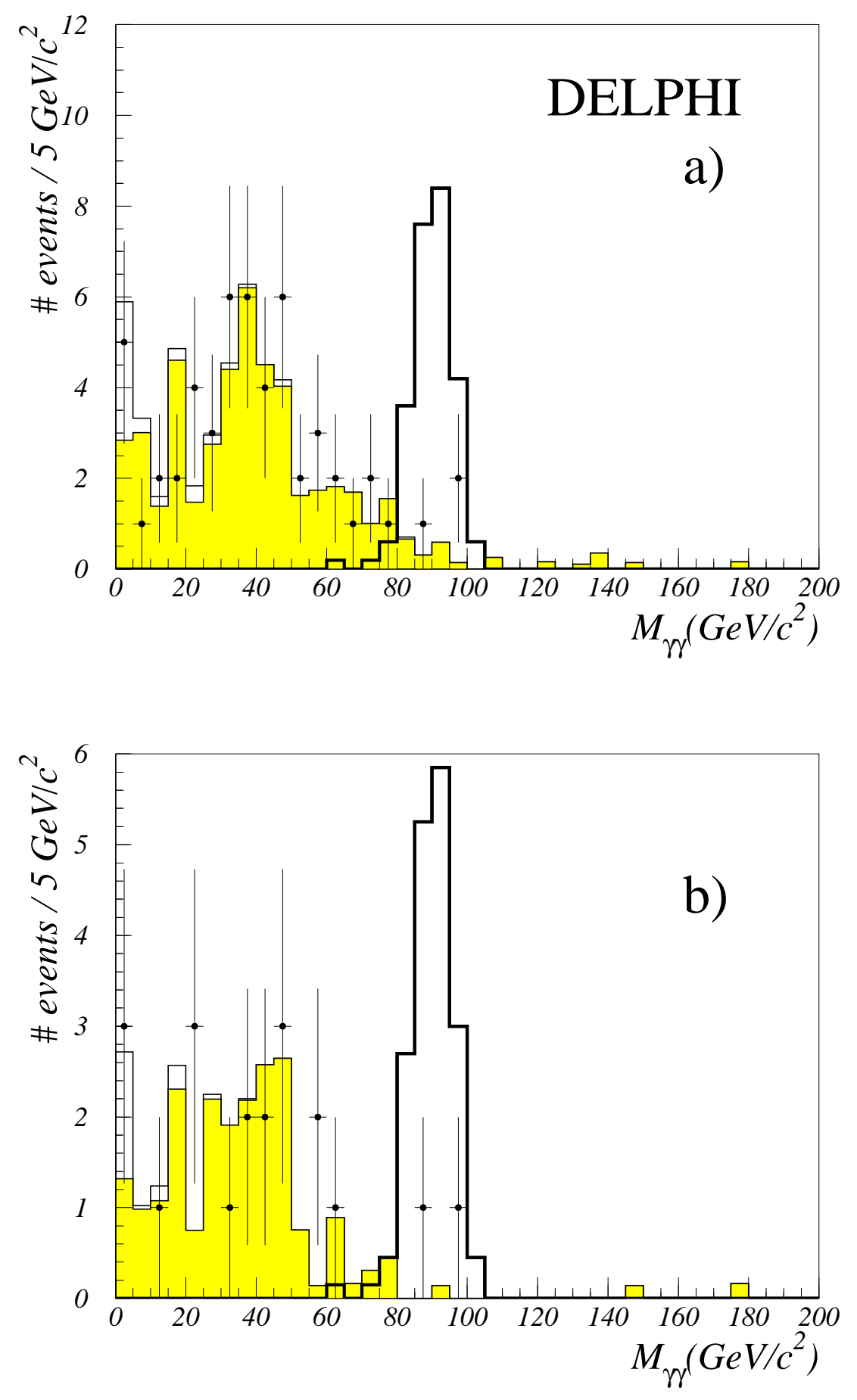

Figure 2: Invariant $\gamma \gamma$ mass at selection level 2 (a) and 3 (b) of the $q \bar{q} \gamma \gamma$ and $b \bar{b} \gamma \gamma$ analyses. All the data analysed are shown by dots and the shaded histograms represent the total backgrounds. The main background contribution is $e^{+} e^{-} \rightarrow Z^{0 *} / \gamma^{*} \rightarrow q \bar{q} \gamma \gamma$. The darker lines represent signals for a $90 \mathrm{GeV} / c^{2}$ Higgs, with arbitrary normalization. 

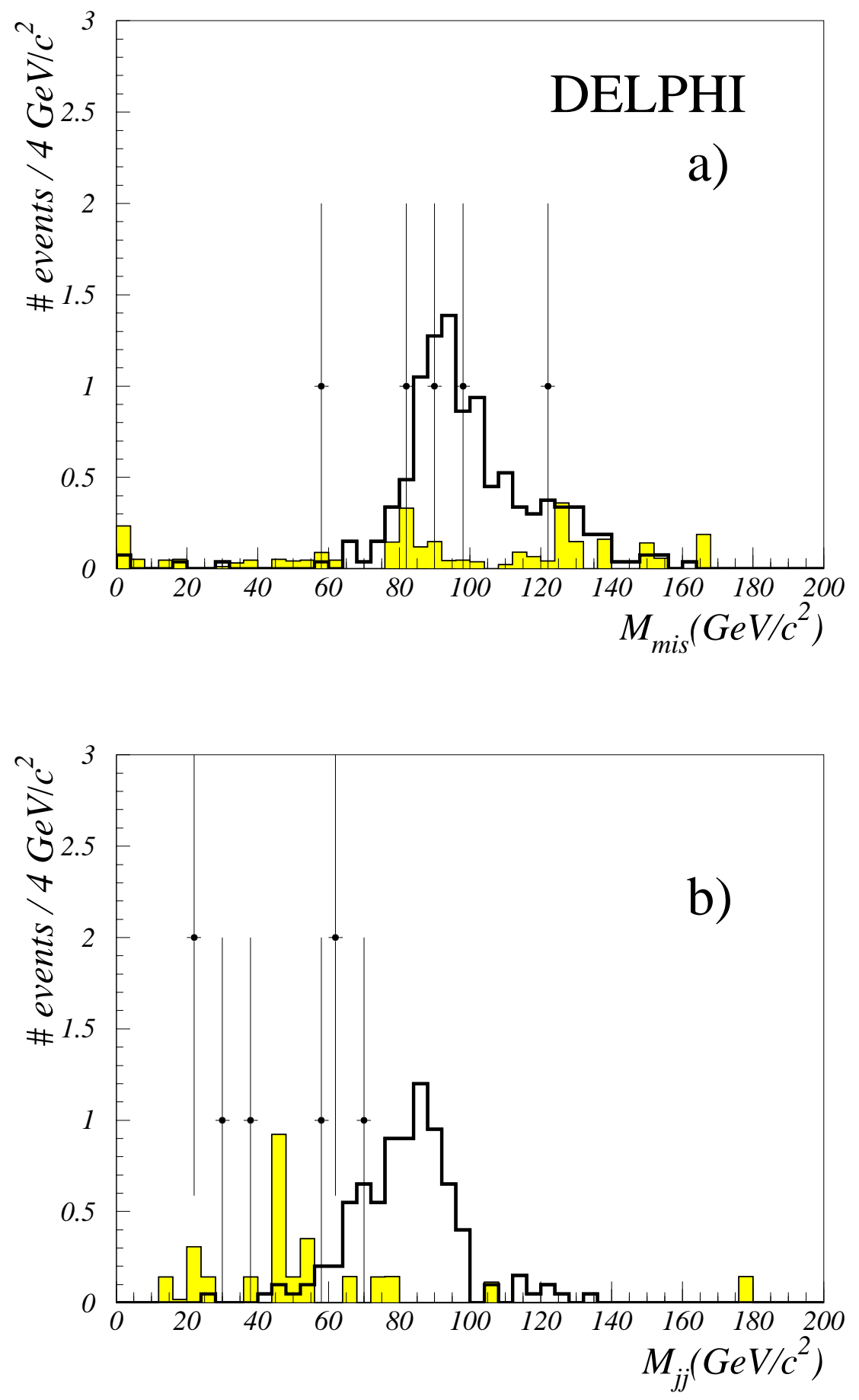

Figure 3: Reconstructed missing mass (a) and invariant jet-jet mass (b) for the two topologies with 4 photons, $\gamma \gamma \gamma \gamma$ and $q \bar{q} \gamma \gamma \gamma \gamma$ respectively. The dots represent the data selected for all the analysed samples. The shaded areas represent the expected standard model background and the darker lines the expectations from $h^{0} A^{0} \rightarrow h^{0} h^{0} Z^{0}$ signals with arbitrary normalization. 


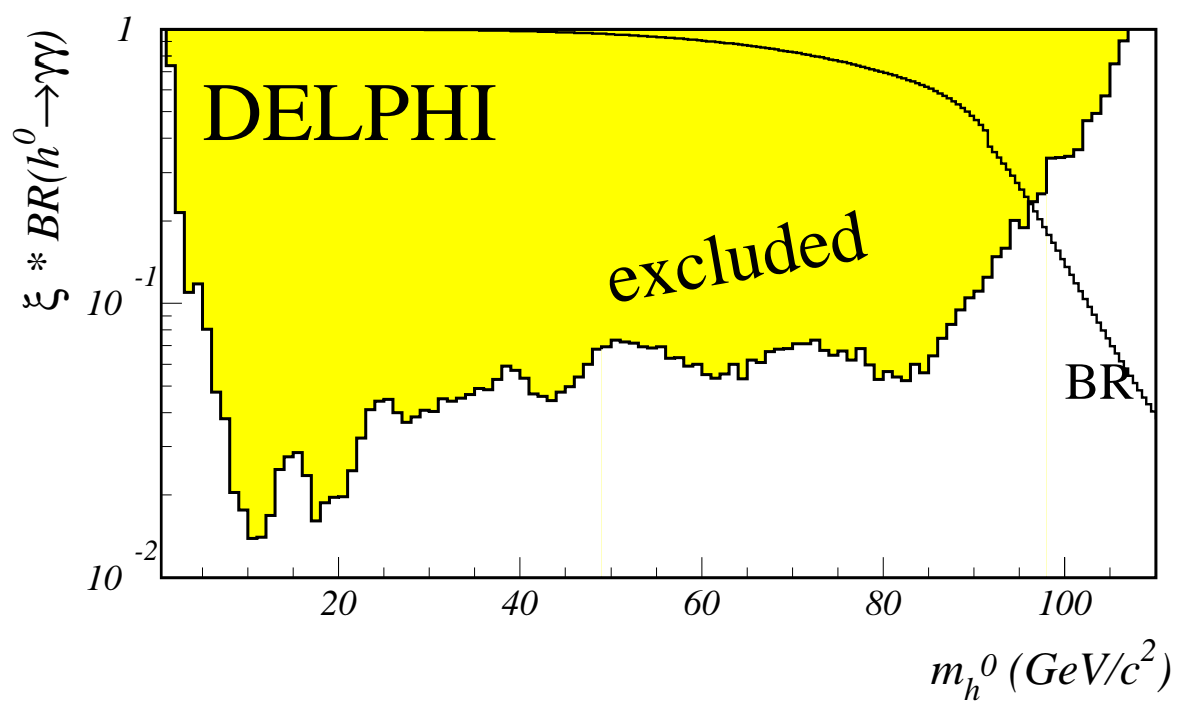

Figure 4: 95\% CL excluded region in the $\left(m_{h^{0}}, \sigma\left(h^{0} Z^{0}\right) / \sigma\left(H Z^{0}\right)_{(S M)} \cdot \operatorname{BR}\left(h^{0} \rightarrow \gamma \gamma\right)\right)$ plane, obtained from the analysis of $q \bar{q} \gamma \gamma$ and $\nu \bar{\nu} \gamma \gamma$. The $\operatorname{BR}\left(h^{0} \rightarrow \gamma \gamma\right)$ computed in [1], for potential A and $\sin ^{2} \delta=1$, is also shown.

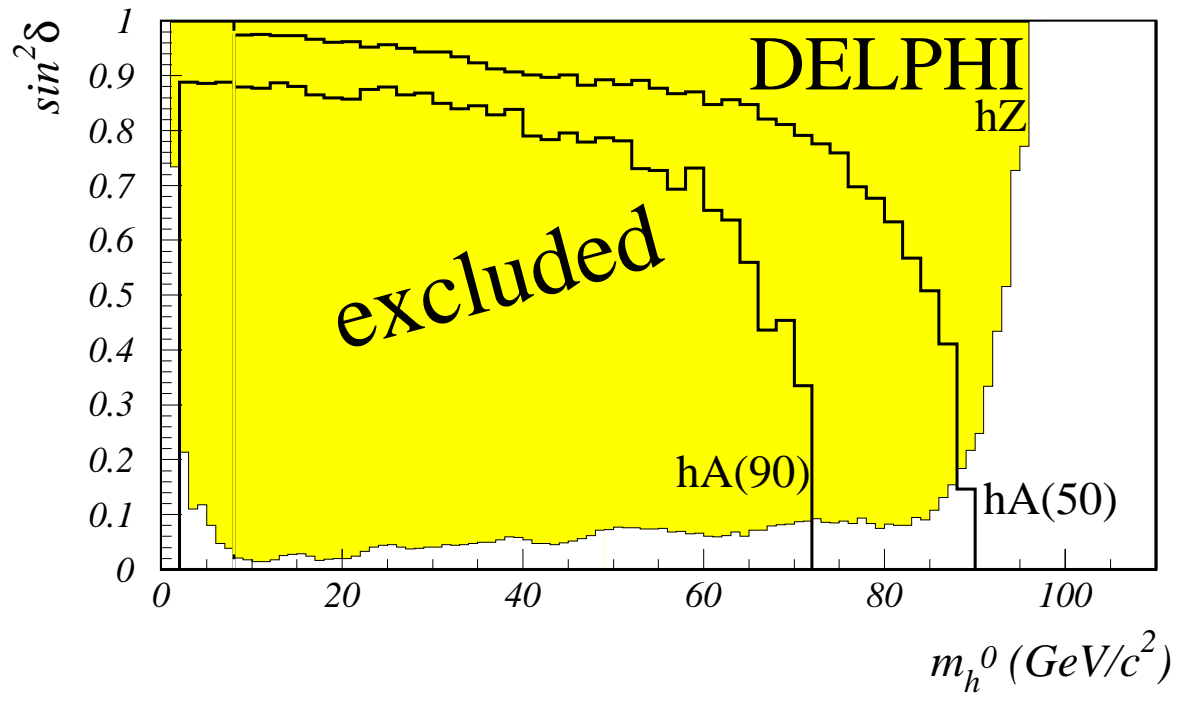

Figure 5: 95\% CL excluded region in the plane $\left(m_{h^{0}}, \sin ^{2} \delta\right)$, obtained from the Higgs-strahlung final states for potential A of 2HDM fermiophobic limit. Also shown are the regions excluded by the associated production process for $m_{A^{0}}=50 \mathrm{GeV} / c^{2}$ and $m_{A^{0}}=90 \mathrm{GeV} / c^{2}$. The $\mathrm{BR}\left(h^{0} \rightarrow \gamma \gamma\right)$ values computed in [1] were used. 

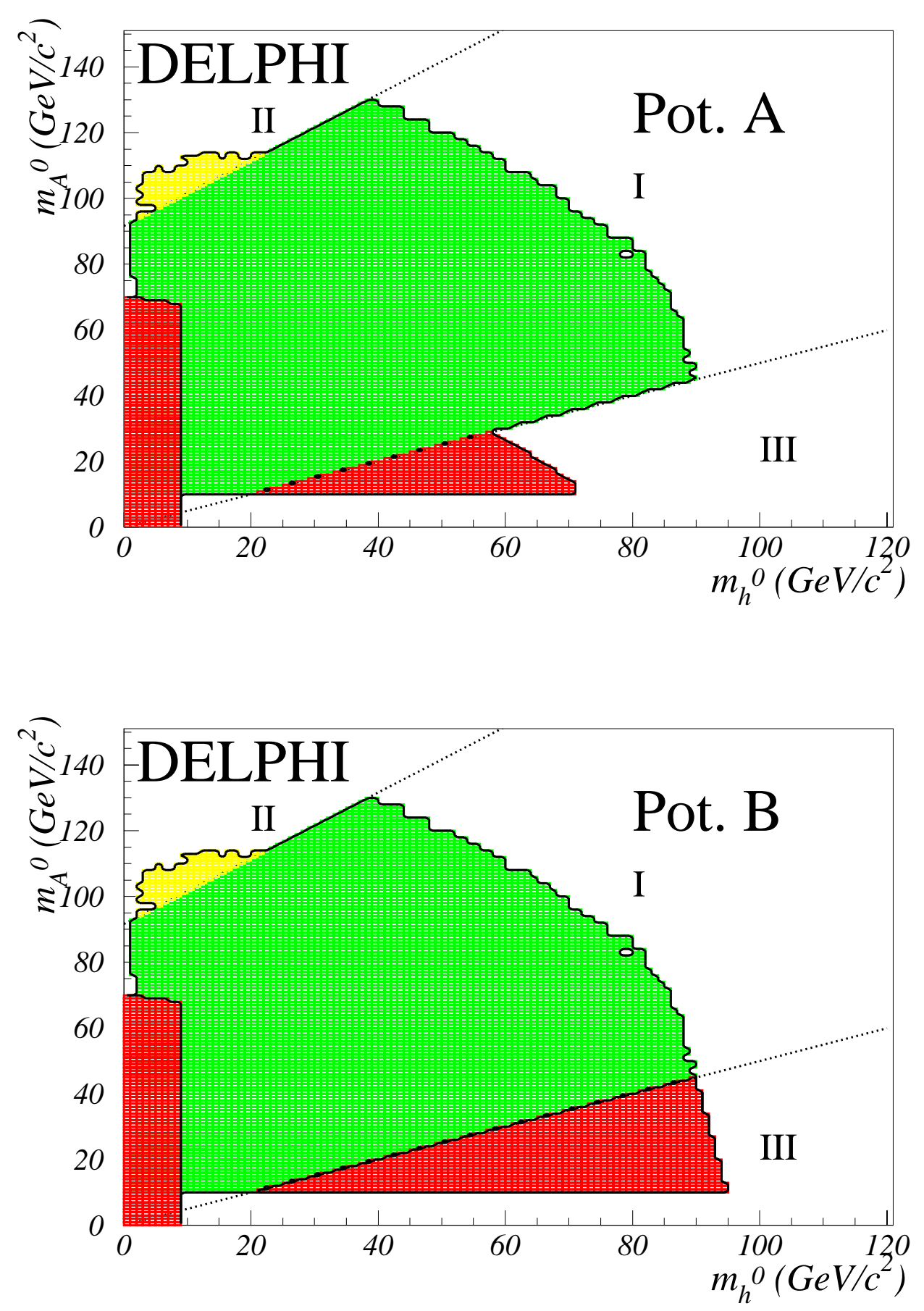

Figure 6: $95 \%$ CL excluded region in the plane $\left(m_{h^{0}}, m_{A^{0}}\right)$, in the framework of Potential A (upper plot) and of Potential B (lower plot). The exclusion is valid for all $\delta$ values and is obtained by combining the Higgs-strahlung and the associated production processes. Region I corresponds to the decay modes $h^{0} \rightarrow \gamma \gamma$ and $A^{0} \rightarrow b \bar{b}$ (or $A^{0}$ long-lived, for Potential A). Region II corresponds to $A^{0} \rightarrow h^{0} Z^{0}$, from the Higgs-strahlung and the two final states with 4 photons. Region III corresponds to $h^{0} \rightarrow A^{0} A^{0}$ and $A^{0} \rightarrow b \bar{b}$ (taken from ref. [8]). For Potential A and very small $\delta$ values, $A^{0}$ is stable and the limit for all $\delta$ comes from the $Z^{0}$ invisible width measurement. The dark band in the low $m_{h^{0}}$ $\left(<9 \mathrm{GeV} / c^{2}\right)$ region represents the limit from the total $Z^{0}$ width. 